Proceedings of the Space Nuclear Conference 2007

Boston, Massachusetts, June 24-28, 2007

Paper 2018

\title{
Can the Equivalent Sphere Model Approximate Organ Doses in Space Radiation Environments?
}

\author{
Zi-Wei Lin \\ Mail Stop VP62, NSSTC, 320 Sparkman Drive, Huntsville, AL 35805 \\ Tel: 256-961-7545; Fax: 256-961-7215; Email: linzx@nsstc.uah.edu
}

\begin{abstract}
In space radiation calculations it is often useful to calculate the dose or dose equivalent in blood-forming organs (BFO), the skin or the eye. It has been customary to use a $5 \mathrm{~cm}$ equivalent sphere to approximate the BFO dose. However, previous studies have shown that a $5 \mathrm{~cm}$ sphere gives conservative dose values for BFO. In this study we use a deterministic radiation transport with the Computerized Anatomical Man model to investigate whether the equivalent sphere model can approximate organ doses in space radiation environments. We find that for galactic cosmic rays environments the equivalent sphere model with an organ-specific constant radius parameter works well for the BFO dose equivalent and marginally well for the BFO dose and the dose equivalent of the eye or the skin. For solar particle events the radius parameters for the organ dose equivalent increase with the shielding thickness, and the model works marginally for BFO but is unacceptable for the eye or the skin. The ranges of the radius parameters are also shown and the BFO radius parameters are found to be significantly larger than $5 \mathrm{~cm}$ in all cases.
\end{abstract}

\section{INTRODUCTION}

Space radiation to astronauts from solar particle events (SPE) and galactic cosmic rays (GCR) is a major hazard for human space explorations, and in space radiation calculations one often needs to calculate the dose or dose equivalent in organs such as BFO, the skin or eyes. The detailed thickness distribution functions of organs can be used for accurate calculations, ${ }^{1,2}$ while sometimes the equivalent sphere model (ESM) with an organspecific constant radius parameter is used to estimate the organ dose in order to save the computation time. It has been customary to use a $5 \mathrm{~cm}$ equivalent sphere to simulate the $\mathrm{BFO}$ dose. However, previous studies ${ }^{1-4}$ have demonstrated that a $5 \mathrm{~cm}$ sphere gives higher (i.e., conservative) dose values compared with the exact $\mathrm{BFO}$ dose. One study ${ }^{3}$ concluded that a $9 \mathrm{~cm}$ sphere is a reasonable approximation for the $\mathrm{BFO}$ dose or dose equivalent in SPE environments when there is an Aluminum shielding of $2-20 \mathrm{~g} / \mathrm{cm}^{2}$.

In this study we investigate whether the equivalent sphere model with an organ-specific constant radius parameter can approximate dose or dose equivalent values in BFO, the eye or the skin in SPE or GCR enviromments. We use a deterministic radiation transport ${ }^{5}$ with organ geometry from the Computerized Anatomical Man (CAM) model. ${ }^{6}$ Six different SPE environments and seven different GCR environments are used in order to see how much the results depend on the choice of the space radiation environment. Calculations have been performed with and without extra shielding materials surrounding the organs in order to study the dependence of the ESM radius parameters on the thickness of the shielding material. We also give a summary of the ranges of the radius parameters of each organ in SPE and GCR environments so that one can easily see in which cases the equivalent sphere model works.

\section{METHODS}

For the following cumulative thickness distribution function of an organ:

$$
F(t) \equiv \int_{0}^{t} f\left(t^{\prime}\right) d t^{\prime}
$$

the thickness distribution function is given by

$$
f(t) \equiv \frac{d F(t)}{d t}
$$

and it is already normalized. In Fig. 1 the curves with circles represent the cumulative thickness distribution function $F(t)$ of BFO (solid), the eye (dashed) and the skin (dot-dashed) from the CAM model. ${ }^{7}$ The curves 
without circles, obtained numerically from the cumulative thickness distribution functions, represent the corresponding organ thickness distribution functions multiplied by the thickness, i.e., $t f(t)$. In this way, the area under the curves without circles is proportional to the probability of the corresponding thickness range.

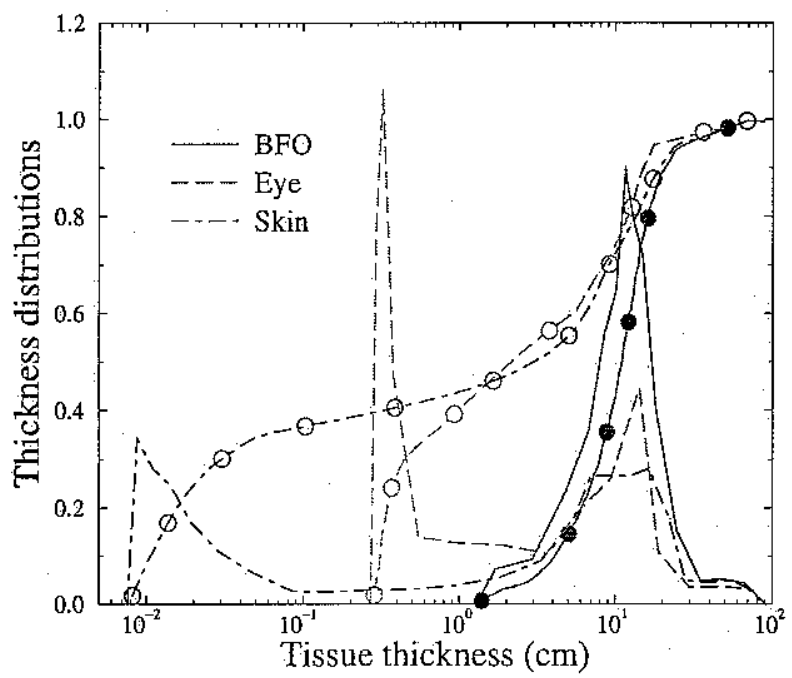

FIG. 1: Thickness distributions for the skin, the eye and BFO from the CAM model. Curves with circles represent the cumulative distribution functions and curves without circles represent the thickness distribution functions multiplied by the thickness.

We use a version of the deterministic radiation transport HZETRN ${ }^{5}$. The six SPE environments used in this study are the Aug. 4, 1972, Aug. 12, 1989, Sept. 29, 1989, Oct. 19, 1989, Mar. 23, 1991 events, ${ }^{8}$ and the Jan. 20, 2005 event, ${ }^{9}$ respectively. For the Jan. 20, 2005 event a parameterized primary alpha particle $\mathrm{flux}^{10}$ is also included. The seven GCR environments are taken from those implemented in HZETRN, ${ }^{5}$ and they are the solar maximum environments in 1958-1959, 1970-1971, 1981-1982, 1989, and the solar minimum environments in 1965, 1977 and 1986-1987. The dose and dose equivalent values are calculated as a function of the thickness of a water slab that is behind a shielding material of a given thickness, and the ICRP $60^{11}$ quality factor is used for the conversion to the dose equivalent if not specified otherwise. For example, the dose equivalent as a function of water depth when there is no extra shielding material is showr in Fig. 2 for the SPE environments and in Fig. 3 for the GCR environments.

We use water to simulate tissue and calculate the organ close and dose equivalent according to the following: ${ }^{1}$

$$
D_{i}=\int \mathcal{D}(t) f_{i}(t) d t, \quad H_{i}=\int H(t) f_{i}(t) d t
$$

where $i$ labels the different organ, and $D(t)$ and $H(t)$ represents the dose and dose equivalent, respectively, as a function of water depth $t$ in a space radiation environment, which for these 1-dimensional slab calculations is considered to be uni-directional instead of being isotropic. We calculate the dose-depth curves $D(t)$ and $H(t)$ both with and without a slab shielding material of thickness $t_{s}$ in front of the semi-infinite water slab of thickness $t$. Because the deterministic radiation transport we use here assumes the straight-ahead approximation, i.e., the nuclear fragments do not change their directions relative to the projectile nucleus, the organ dose calculated using $D(t)$ or $H(t)$ behind a slab shielding material of thickness $t_{s}$ is equivalent to the dose of the organ that is surrounded by a shell of the shielding material of thickness $t_{s}$ with the shell having a large inner radius.

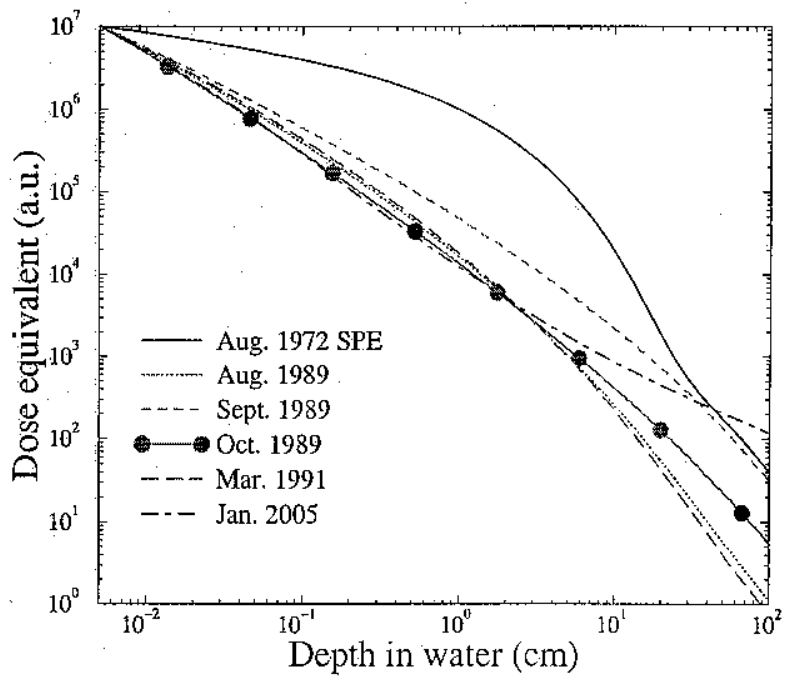

FIG. 2: Dose equivalent as a function of water depth in different SPE environments with no extra shielding.

The ESM radius parameters in the equivalent sphere model, $R_{i}^{D}$ for the dose and $R_{i}^{H}$ for the dose equivalent of organ $i$, are calculated by finding the water depth where the dose or dose equivalent is equal to the exact organ values calculated from $\mathrm{Eq}$. (3), i.e.,

$$
D\left(R_{i}^{D}\right)=D_{i}, \quad H\left(R_{i}^{H}\right)=H_{i} .
$$

Eqs. (3-4) show that the radius parameters are determined by the shapes of the dose-depth curves but not by their overall magnitudes, and this is the reason that we have renormalized the dose-depth curves to arbitrary units in Fig. 2-3 in order to better show the difference in their shapes. 


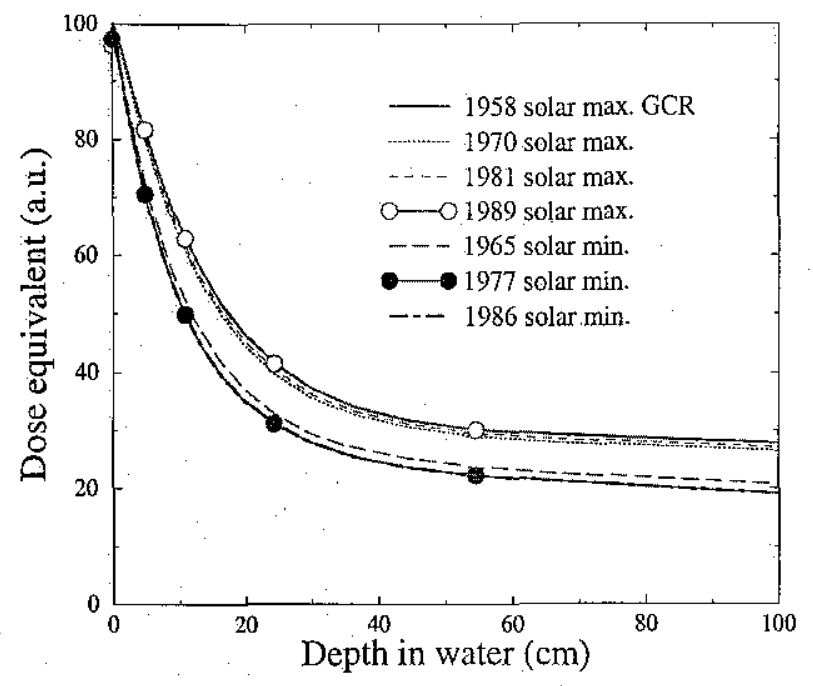

FIG. 3: Dose equivalent as a function of water depth in different GCR environments with no extra shielding.

\section{RADIUS PARAMETERS OF ORGANS WITH NO EXTRA SHIELDING}

First we have calculated the ESM radius parameters for the organ dose and dose equivalent when there is no extra shielding material. These radius parameters for each SPE environment are shown in Table I. We find that the BFO radius parameters for different SPEs for both the dose equivalent and the dose are within about $25 \%$ of each other. The radius parameters of the eye for different SPEs are within a factor of 2 of each other. However, the SKIN radius parameters for the Aug. 1972 SPE are far from those for the other SPEs, as much as larger by a factor of 8 . This can be understood qualitatively from the dose-depth curves shown in Fig. 2. As the dose equivalent values of SPEs decrease by orders of magnitude from $0.005 \mathrm{~cm}$ to a few $\mathrm{cm}$ in water, the total skin dose or dose equivalent mostly comes from that at small thicknesses, say, below $1 \mathrm{~g} / \mathrm{cm}^{2}$, which make up roughly about half of the thickness distribution of the skin. Therefore the total skin dose is about half of the dose value at a small depth scale that is representative of the skin thickness distribution at small thicknesses. As a result, the skin radius parameter is roughly determined by the water depth where the dose at that representative small depth scale decreases by a factor of 2 . We can see that the dose-depth curve of the Aug. 1972 SPE has a much smaller slope at small thicknesses than those of the other SPEs, and this leads to larger radius parameters for the skin in the Aug. 1972 SPE environment.

The ESM radius parameters for each GCR environment are shown in Table $\mathrm{II}$. We find that the radius parameters of each organ are much larger than the corre-
TABLE I: Radius parameters (in $\mathrm{cm}$ ) for the organ dose equivalent $(\mathrm{H})$ and organ dose (D) in SPE environments when there is no shielding material. The SPE number 1 to 6 represents the Aug. 1972, Aug. 1989, Sept. 1989, Oct. 1989, Mar. 1991, and Jan. 2005 event, respectively.

\begin{tabular}{|c|c|c|c|c|c|c|}
\hline SPE Event \# & 1 & 2 & 3 & 4 & 5 & 6 \\
\hline$R_{\text {BFO }}^{\mathrm{H}}$ & 6.8 & 6.6 & 7.4 & 7.1 & 6.4 & 7.7 \\
\hline$R_{\text {Eye }}^{\mathrm{H}}$ & 1.1 & 0.68 & 0.77 & 0.69 & 0.67 & 0.69 \\
\hline$R_{\text {Skin }}^{\mathrm{H}}$ & 0.21 & 0.038 & 0.043 & 0.035 & 0.039 & 0.035 \\
\hline$R_{\mathrm{BFO}}^{\mathrm{D}}$ & 6.9 & 6.8 & 7.7 & 7.2 & 6.6 & 8.0 \\
\hline$R_{\text {Eye }}^{\mathrm{D}}$ & 1.2 & 0.74 & 0.87 & 0.76 & 0.74 & 0.78 \\
\hline$R_{\text {Skin }}^{\mathrm{D}}$ & 0.41 & 0.055 & 0.070 & 0.049 & 0.057 & 0.049 \\
\hline
\end{tabular}

sponding value in the SPE environments, especially for the eye and the skin. Furthermore, the radius parameters in solar maximum GCR environments are larger than those in solar minimum GCR environments. For the organ dose equivalent, the radius parameters for different GCR environments are close to each other for each of the three organs, especially for BFO. However, the radius parameters for the organ dose for different GCR environments are close to each other only for BFO.

TABLE II: Radius parameters (in $\mathrm{cm}$ ) for the organ dose equivalent $(\mathrm{H})$ and organ dose (D) in GCR environments when there is no surrounding material. The GCR number 1 to 4 represents the solar maximum environments in 1958 $1959,1970-1971,1981-1982$, and 1989 , respectively, while number 5 to 7 represents the solar minimum environments in 1965, 1977 and 1986-1987, respectively.

\begin{tabular}{|c|c|c|c|c||c|c|c|}
\hline GCR Environment \# & 1 & 2 & 3 & 4 & 5 & 6 & 7 \\
\hline$R_{\mathrm{BFO}}^{\mathrm{H}}$ & 10.7 & 10.6 & 10.6 & 10.7 & 10.3 & 10.2 & 10.2 \\
\hline$R_{\mathrm{Eye}}^{\mathrm{H}}$ & 4.8 & 4.7 & 4.7 & 4.8 & 4.1 & 3.9 & 3.9 \\
\hline$R_{\text {Skin }}^{\mathrm{H}}$ & 5.5 & 5.3 & 5.4 & 5.5 & 4.5 & 4.3 & 4.3 \\
\hline$R_{\mathrm{BFO}}^{\mathrm{D}}$ & 11.9 & 11.5 & 11.6 & 11.8 & 11.0 & 11.0 & 11.0 \\
\hline$R_{\mathrm{Eve}}^{\mathrm{D}}$ & 12. & 9.3 & 10. & 11. & 5.6 & 5.1 & 5.1 \\
\hline$R_{\text {Skin }}^{\mathrm{D}}$ & 18. & 14. & 15. & 17. & 7.3 & 6.6 & 6.6 \\
\hline
\end{tabular}

Because the dose-depth curves in GCR environments change much more slowly with water depth compared with those in SPE environments, they have much smaller curvatures. Consequently the ESM radius parameters of each organ in GCR environments are significantly larger than those in SPE environments, and they are also on the same order of magnitude as the average thickness value of the organ, which is about $13 ., 6.8$ and $7.5 \mathrm{~cm}$, respectively, for $\mathrm{BFO}$, the eye and the skin from the CAM model. Also, a solar maximum GCR environment has about the same particle flux at high energies, above about $10 \mathrm{GeV}$ per nucleon, but far less lower energy particles than a solar minimum GCR environment. Consequently the dose equivalent-depth curves for solar 
maximum GCR environments decrease more slowly than those for solar minimum GCR environments, as shown in Fig. 2, and this leads to somewhat larger values for the ESM radius parameters in solar maximum GCR environments.

\section{EFFECTS OF SHIELDING MATERIALS ON THE RADIUS PARAMETERS OF ORGANS}

Organ doses are often calculated with space radiation environments that have been modified by surrounding materials, such as a spacesuit, a spacecraft or the habitat. To investigate how the ESM radius parameters change in the presence of such shielding, we have calculated the dose and dose equivalent as a function of water depth behind a shielding material. The slab shielding material is either water or Aluminum at thickness between 0 and $20 \mathrm{~g} / \mathrm{cm}^{2}$, and it is positioned in front of the water slab that simulates tissue. We then determine the radius parameters using Eqs. (3-4).

The radius parameters for the Aug. 1972 and the Oct. 1989 SPEs are shown in Fig. 4 for BFO, in Fig. 5 for the eye, and in Fig. 6 for the skin, as a function of the areal density of the water shielding. We see that all the radius parameters increase with the thickness of the shielding material. Furthermore, the difference in the radius parameters between the two SPE environments can be significant. The $\mathrm{BFO}$ radius parameters increase from about $7 \mathrm{~cm}$ when there is no shielding material to up to $10.5 \mathrm{~cm}$ when there is a shielding material of $20 \mathrm{~g} / \mathrm{cm}^{2}$. This increase is only up to $50 \%$, therefore one may use the equivalent sphere model with a constant radius parameter to simulate the $\mathrm{BFO}$ dose or dose equivalent in SPE environments in situations where an error on the order of a factor of 2 (see Fig. 2) can be tolerated. On the other hand, the increase with the shielding thickness is much stronger for the eye (by a factor of up to 5) and the strongest for the skin radius parameters (by a factor of up to 100). This is mainly due to the large curvatures of the dose-depth curves in SPE environments. As a result, the dose behind a finite shielding material decreases much more slowly with water depth, and the smaller curvature of the dose-depth curve leads to a larger radius parameter. Figs. 4-6 show that the equivalent sphere model with an organ-specific constant radius parameter works marginally for BFO but cannot reliably simulate the dose or dose equivalent of the eye or the skin in SPF environments.

The radius parameters for both the 1977 solar minimum and the 1989 solar maximum GCR environments are shown in Fig. 7 for BFO, in Fig. 8 for the eye, and in Fig. 9 for the skin, as a function of the areal density of the water shielding. For the radius parameters for the organ dose equivalent, we find that the ranges of change with the shielding thickness in GCR environments are much smaller than those in SPE environments.

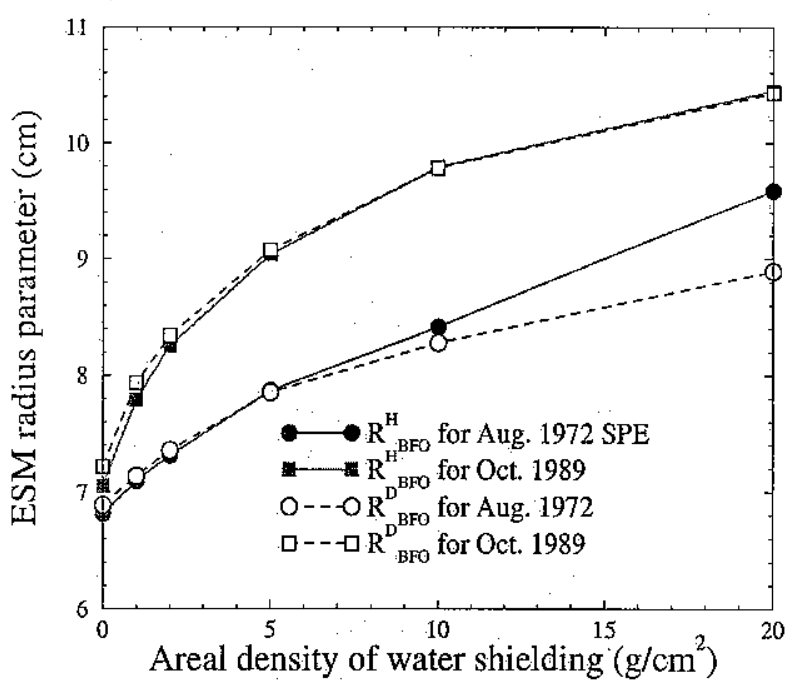

FIG. 4: The radius parameters for BFO in two SPE environments as a function of the areal density of the water shielding.

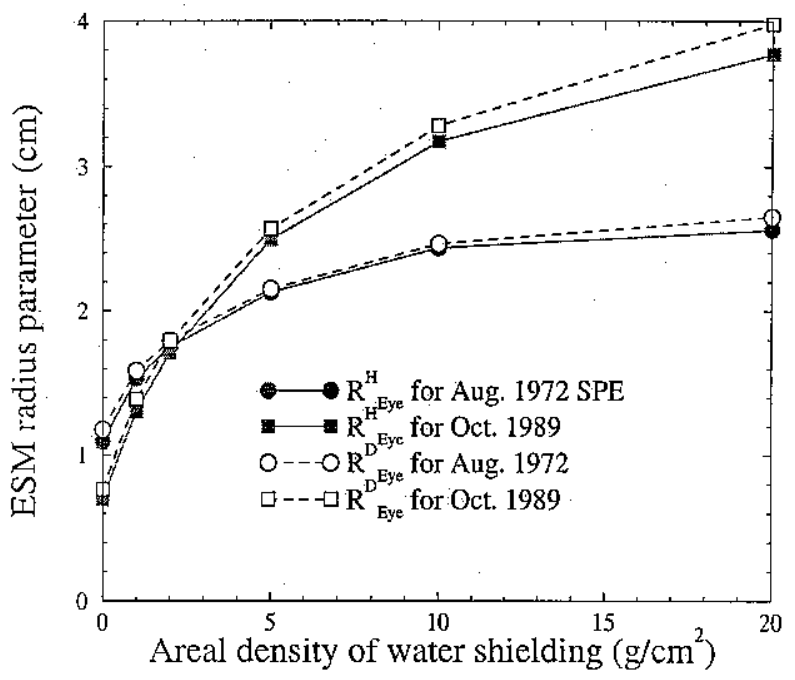

FIG. 5: The radius parameters for the eye in two SPE environments as a function of the areal density of the water shielding.

Furthermore, the radius parameters for the organ dose equivalent in solar minimum GCR environments are essentially the same as those in solar maximum GCR enviromments when there is a shielding material of about $20 \mathrm{~g} / \mathrm{cm}^{2}$. Fig. 7 also shows the radius parameters for the BFO dose equivalent, when the older ICRP26 quality factor $^{12}$ is used (dot-dashed curves), and we see that the difference between the ICRP26 and ICRP60 quality factors has little effect on the $\mathrm{BFO}$ radius parameters. We 


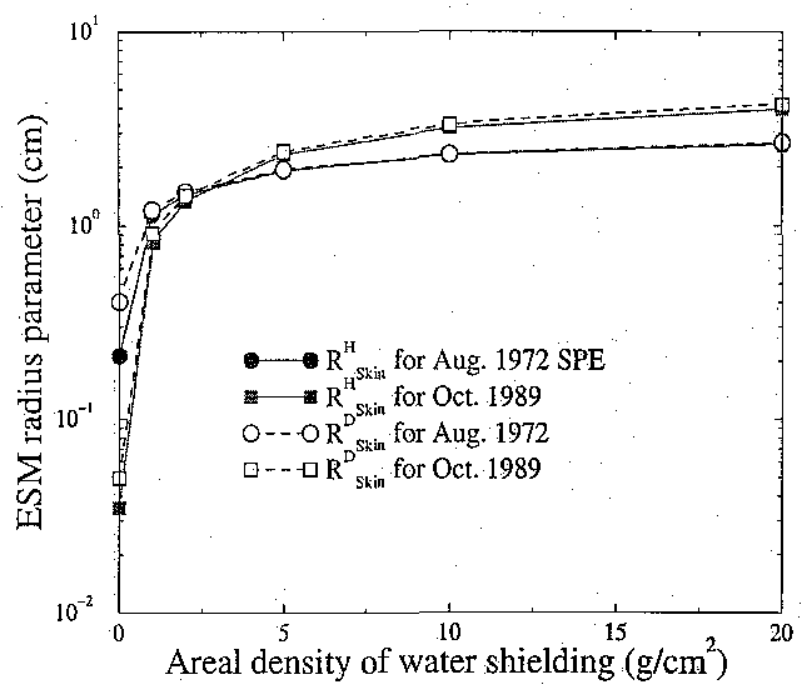

FIG. 6: The radius parameters for the skin (in the logarithmic scale) in two SPE environments as a function of the areal density of the water shielding.

find that this is also true for the radius parameters of the eye and the skin. Overall, the radius parameters for the organ dose equivalent are found to be approximately from 10 to $11 \mathrm{~cm}$ for the $\mathrm{BFO}, 3.7$ to $4.8 \mathrm{~cm}$ for the eye, and 3.5 to $5.6 \mathrm{~cm}$ for the skin in GCR environments. Therefore the equivalent sphere model with a constant radius parameter works well for the $\mathrm{BFO}$ dose equivalent and marginally well for the eye or skin dose equivalent in GCR environments.

The ESM radius parameters for the organ dose in GCR environments, as shown in Figs. 7-9 as well as Table II, can be quite different from the corresponding radius parameters for the organ dose equivalent. This difference is much smaller in SPE environments where heavy ion contributions to the organ dose equivalent is negligible. For the organ dose, the range of the ESM radius parameters is approximately from 10 to $13 \mathrm{~cm}$ for the BFO, 2.8 to $12 \mathrm{~cm}$ for the eye, and 2.3 to 19 $\mathrm{cm}$ for the skin. Therefore the equivalent sphere model with a constant radius parameter works marginally for the BFO dose but not for the eye or skin dose in GCR environments.

Fig. 10 shows the BFO radius parameters with water or Aluminum shielding in the Oct. $1989 \mathrm{SPE}$ (curves with symbols) or the 1977 solar minimum GCR envirorment (curves without symbols). We find that the difference in the radius parameters between water and Aluminum shielding is typically smaller than the range of the radius parameters over the studied shielding thicknesses, especially for the radius parameters for the dose equivalent. Fig. 10 and Fig. 4 are also consistent with an earlier study ${ }^{3}$ which concluded that a $9 \mathrm{~cm}$ sphere is

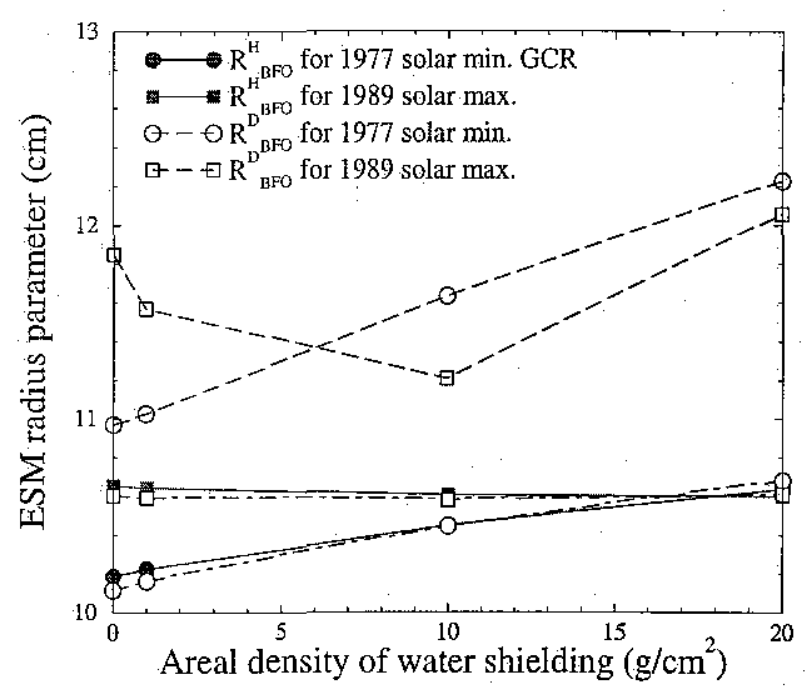

FIG. 7: The radius parameters for BFO in GCR environments as a function of the areal density of the water shielding surrounding the organ. The dot-dashed curves represent the radius parameters for the BFO dose equivalent when the ICRP26 quality factor is used.

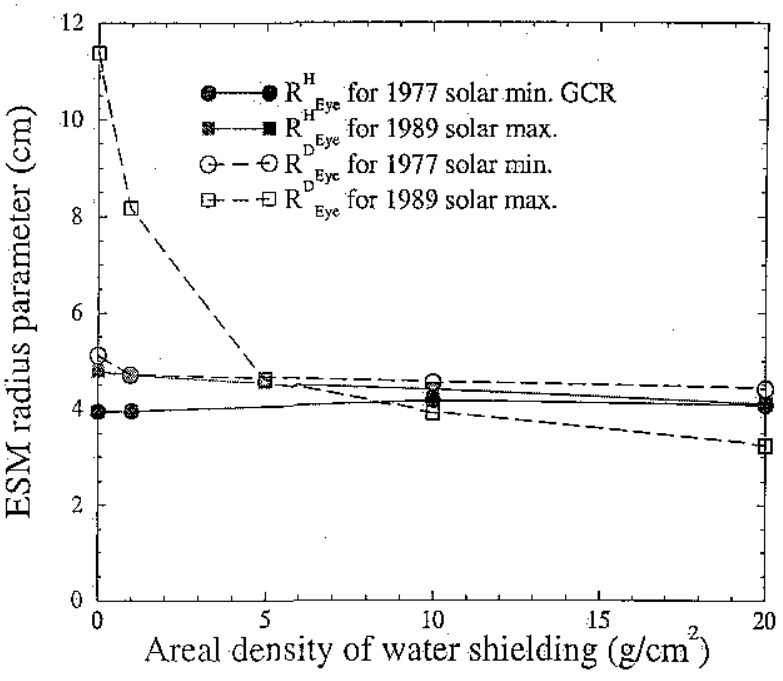

FIG. 8: The radius parameters for the eye in GCR environments as a function of the areal density of the water shielding.

a reasorable approximation for the $\mathrm{BFO}$ dose or dose equivalent in SPE environments when there is an Aluminum shielding of $2-20 \mathrm{~g} / \mathrm{cm}^{2}$.

A summary of the ranges of the ESM radius parameters for two SPE and two GCR environments for each of the three organs is provided in Table III. When the change of the dose or dose equivalent over a given range 


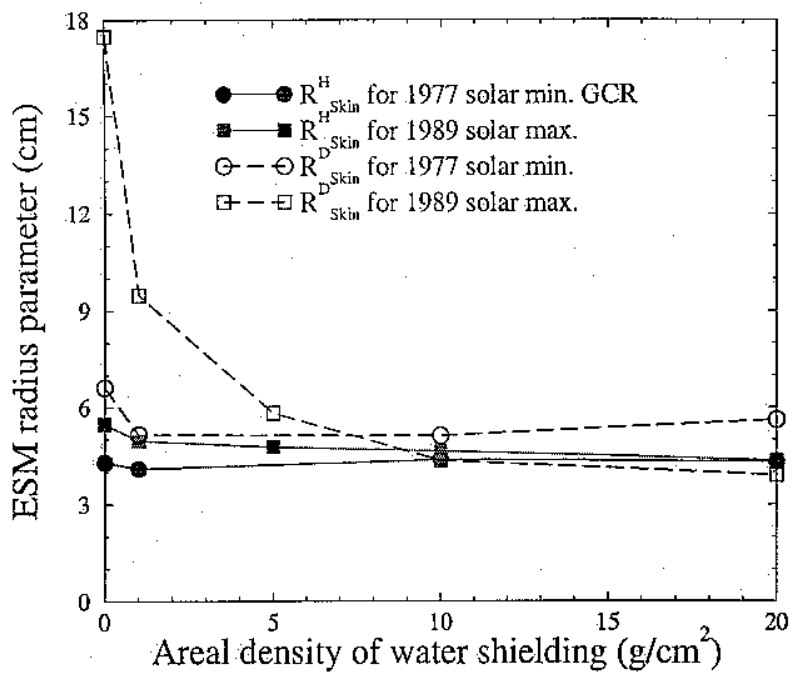

FIG. 9: The radius parameters for the skin in GCR environments as a function of the areal density of the water shielding.

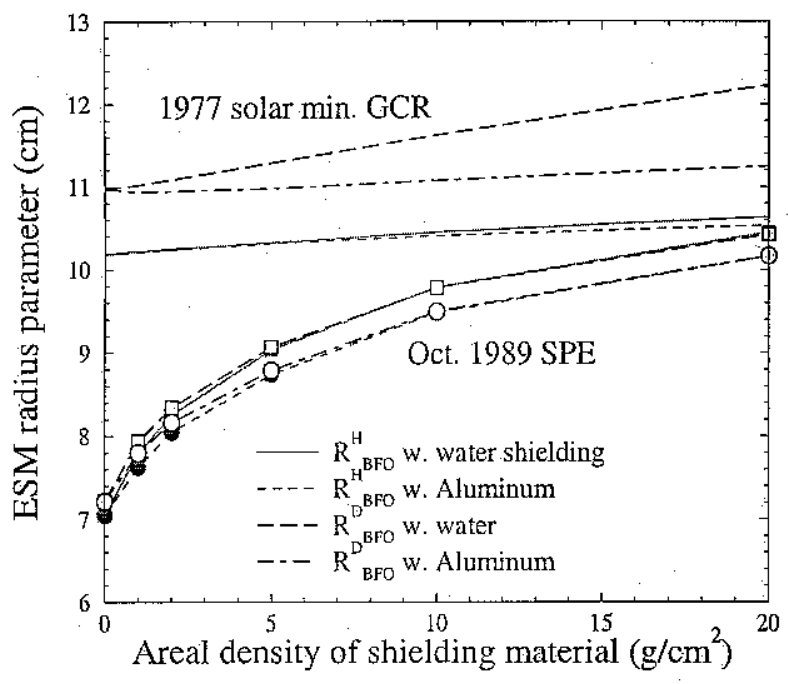

FIG. 10: The BFO radius parameters as a function of the areal density of the water or Aluminum shielding.

is small, the equivalent sphere model with a constant radius parameter within that range can provide a reasonable approximation of the organ dose or dose equivalent.

\section{DISCUSSION}

We see that all the radius parameters for the organ dose equivalent are smaller than the average thickness value of the organ. This is because the dose equivalentdepth curves in both GCR and SPE environments are
TABLE III: Ranges of the ESM radius parameters (in $\mathrm{cm}$ ) for the organ dose equivalent $(H)$ and organ close (D) in two GCR and two SPE environments with a water or Aluminum shielding of thickness between 0 and $20 \mathrm{~g} / \mathrm{cm}^{2}$.

\begin{tabular}{|c|c|c|}
\hline & 1977 and 1989 GCRs & Aug.'72 and Oct.'89 SPEs \\
\hline$R_{\text {BFO }}^{\mathrm{H}}$ & $10-11$ & $6.8-10.5$ \\
\hline$R_{\mathrm{Eye}}^{\mathrm{H}}$ & $3.7-4.8$ & $0.69-3.8$ \\
\hline$R_{\mathrm{Skin}}^{\mathrm{H}}$ & $3.5-5.6$ & $0.035-4.0$ \\
\hline$R_{\mathrm{BFO}}^{\mathrm{D}}$ & $10-13$ & $6.9-10.5$ \\
\hline$R_{\mathrm{Eye}}^{\mathrm{D}}$ & $2.8-12$ & $0.76-4.0$ \\
\hline$R_{\text {Skin }}^{\mathrm{D}}$ & $2.3-19$ & $0.049-4.2$ \\
\hline
\end{tabular}

mostly convex over the range of water depths that is important for the organ dose equivalent, i.e., the curvatures are non-negative. Jansen's inequality states that, for a real convex function $\phi(x)$, one has

$$
\phi(E\{x\}) \leq E\{\phi(x)\},
$$

where $E\{Y(x)\}$ represents the expectation value of $Y(x)$ with respect to a non-negative probability distribution function in variable $x$. Replacing $\phi(x)$ by $H(t)$ leads to

$$
H\left(\bar{t}_{i}\right) \leq H_{i}
$$

where $\bar{t}_{i}$ is the average thickness value of organ $i$. As the dose equivalent-depth curves in both GCR and SPE environments mostly decrease with depth, Eq. (6) means

$$
R_{i}^{\mathrm{F}} \leq \bar{t}_{i}
$$

On the other hand, the radius parameters for the organ dose in GCR environments could be higher than the average thickness value of the organ, as seen from Table II, because sometimes a significant part of the dose-depth curves can be concave. Note that the curvature of the dose equivalent-depth curve matters more to the above inequality equations than the slope of the curve. One can easily see from Eqs. (3-4) that, if the dose equivalent changes linearly with depth over the relevant depth range, one has $R_{i}^{\mathrm{H}}=\bar{t}_{i}$ regardless of the value of the coefficient of the linear term.

We have shown that the equivalent sphere model with an organ-specific constant radius parameter does not work well for either the dose or the dose equivalent of the eye or the skin in SPE environments. In GCR environments it works marginally for the dose equivalent of the eye or the skin but does not work for their doses. One possibility to improve the equivalent sphere model is to use two radius parameters to represent the eye or the skin, because it is clear from Fig. 1 that these two organs are better represented by a superposition of two thickness distribution functions instead of one. 


\section{SUMMARY}

We have used a deterministic radiation transport with the organ geometry from the Computerized Anatomical Man model to study when the equivalent sphere model with an organ-specific constant radius parameter can approximate the organ dose or dose equivalent in space radiation environments. The organ doses have been evaluated with a water or Aluminum shielding of an areal density from 0 to $20 \mathrm{~g} / \mathrm{cm}^{2}$. For the six different $\mathrm{SPE}$ environments, the $\mathrm{BFO}$ radius parameters are approximately between 6.4 and $8.0 \mathrm{~cm}$ in case of no shielding, and they increase with the thickness of the shielding material (by up to $50 \%$ at $20 \mathrm{~g} / \mathrm{cm}^{2}$ ). On the other hand, the radius parameters for the eye or the skin increase sharply with the shielding thickness. Therefore for SPE environments the equivalent sphere model works marginally for BFO but does not work for the estimation of the dose or dose equivalent of the eye or the skin. For the seven different GCR environments, the radius parameters for the dose equivalent are approximately between 10 and $11 \mathrm{~cm}$ for the BFO, 3.7 to 4.8 $\mathrm{cm}$ for the eye, and 3.5 to $5.6 \mathrm{~cm}$ for the skin. The radius parameters are between 10 and $13 \mathrm{~cm}$ for the $\mathrm{BFO}$ dose. Therefore for GCR environments the equivalent sphere model works well for the BFO dose equivalent, marginally well for the dose equivalent of the eye or the skin as well as the BFO dose, but not for the dose of the eye or the skin.

\section{ACKNOWLEDGMENTS}

The author acknowledges the support of the National Space Science \& Technology Center through cooperative agreements NCC8-200 and NNM05AA22A.

\section{REFERENCES}

1. J. L. SHINN, J. W. WILSON, and J. E. NEALY, "Reliability of Equivalent Sphere Model in BloodForming Organ Dose Estimation", NASA TM-4178 (1990).

2. J. W. WILSON, F. A. CUCINOTTA, H. TAI, L. C. SIMONSEN, J. L. SHINN, S. A. THIBEAULT, and M. Y. KIM, "Galactic and Solar Cosmic Ray Shielding in Deep Space", NASA TP-3682 (1997).

3. S. G. BIER, L. W. TOWNSEND, and W. L. MAXSON, "New Equivalent Sphere Approximation for BFO
Dose Estimation: Solar Particle Events", Advances in Space Research, 21, 1777 (1998).

4. J. L. HOFF, L. W. TOWNSEND, and E. N. ZAPP, "Interplanetary Crew Doses and Dose Equivalents: Variations among Different Bone Marrow and Skin Sites", Advances in Space Research, 34, 1347 (2004).

5. J. W. WILSON, F. F. BADAVI, F. A. CUCINOTTA, J. L. SHINN, G. D. BADHWAR, R. SILBERBERG, C. H. TSAO, L. W. TOWNSEND, and R. K. TRIPATHI, "HZETRN: Description of a Free-Space Ion and Nucleon Transport and Shielding Computer Program", NASA TP-3495 (1995).

6. M. P. BILLINGS and W. R. YUCKER, "The Computerized Anatomical Man (CAM) Model", NASA CR-134043 (1973).

7. J. E. NEALY, S. A. STRIEPE, and L. C. SIMONSEN, "MIRACAL: A Mission Radiation Calculation Program for Analysis of Lunar and Interplanetary Missions", NASA TP-3211 (1992).

8. M. A. XAPSOS, J. L. BARTH, E. G. STASSINOPOULOS, S. R. MESSENGER, R. J. WALTERS, G. P. SUMMERS, and E. A. BURKE, "Characterizing Solar Proton Energy Spectra for Radiation Effects Applications", IEEE Transactions on Nuclear Science, 47, 2218 (2000).

9. R. A. MEWALDT, M. D. LOOPER, C. M. S. COHEN, G. M. MASON, D. K. HAGGERTY, M. I. DESAI, A. W. LABRADOR, R. A. LESKE, and J. E. MAZUR, "Solar-Particle Energy Spectra during the Large Events of October-November 2003 and January 2005", Proc. 29th International Cosmic Ray Conference $1,111(2005)$.

10. R.A. MEWALDT, private communication.

11. The 1990 Recommendations of the International Commission on Radiological Protection, ICRP Publication 60, Ann. ICRP 21 (1-3) (1991).

12. Recommendations of the International Commission on Radiological Protection, ICRP Publication 26, Ann. ICRP 1 (3) (1977). 


\title{
Can the Equivalent Sphere Model approximate organ doses in space radiation environments?
}

\author{
Zi-Wei Lin \\ University of Alabama in Huntsville / \\ East Carolina University
}




\section{Overview}

Computation is often much easier if one represents an organ with an equivalent sphere. For blood forming organs (BFO), it has been customary to use a $5 \mathrm{~cm}$ equivalent sphere to simulate its dose.
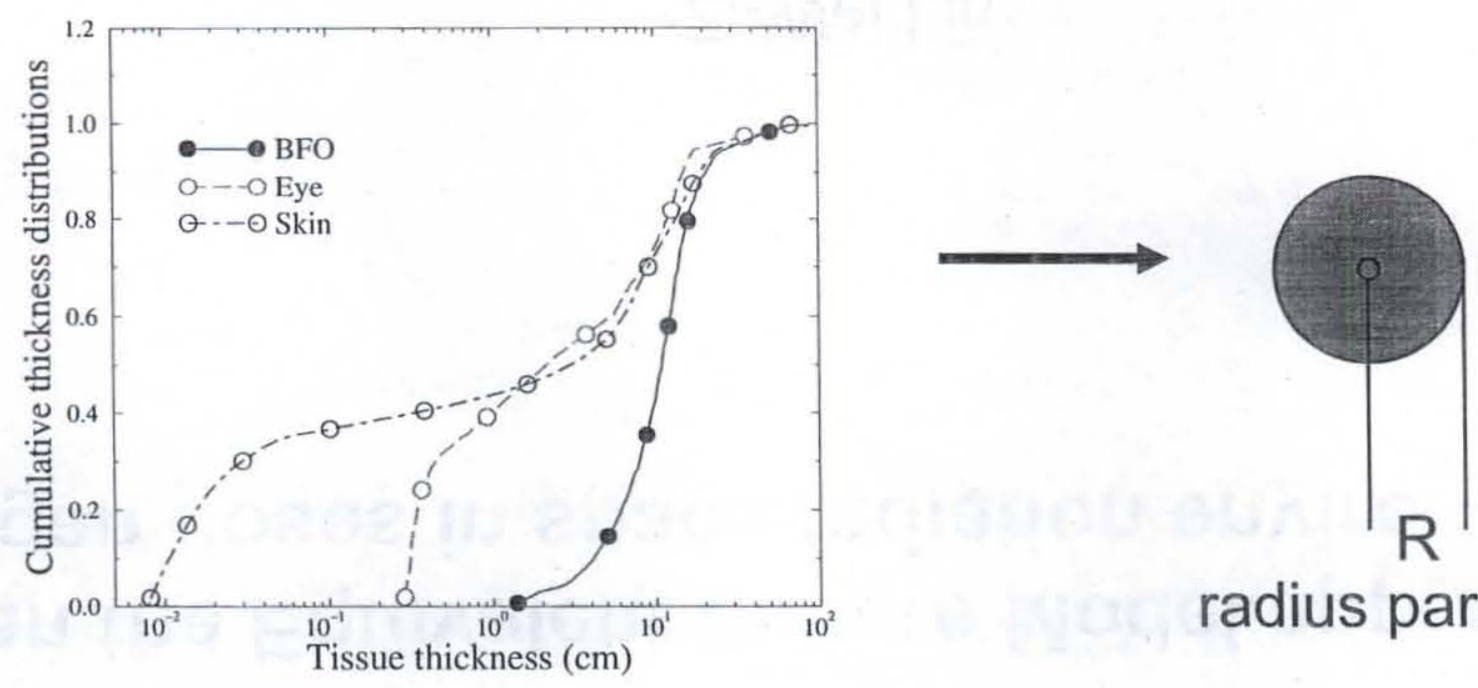

radius parameter

It is well known that a $5 \mathrm{~cm}$ sphere gives conservative dose values. A $9 \mathrm{~cm}$ sphere was found to better approximate BFO doses in SPEs.

Bier, Townsend \& Maxson, Adv. Space Res. 21 (1998) 


\section{Goal}

Investigate the Equivalent Sphere Model (ESM) radius parameters for

-Dose

-Dose equivalent

in

-BFO

-Skin

-Eye

in different space radiation environments

- Solar Particle Events (SPE)

- Galactic Cosmic Ray (GCR)

$\rightarrow$ Where does ESM work and what are the $\mathrm{R}$ (radius) values?

$\rightarrow$ Where does it not work? 


\section{Method}

Take 6 different SPEs and 7 GCRs for radiation environments

Use a 1-D deterministic radiation transport (HZETRN)

to get dose-depth curves $\mathrm{D}(\mathrm{t})$ \& $\mathrm{H}(\mathrm{t}) \quad$ Wilson et al., NASA TP-3495 (1995)

Take skin, eye and BFO thickness distributions $\mathrm{f}(\mathrm{t})$

from the Computerized Anatomical Man (CAM) model

Billings \& Yucker, NASA CR-134043 (1973)

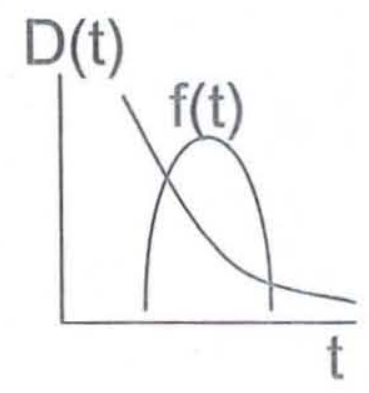

Calculate dose $(\mathrm{D})$ and dose equivalent $(\mathrm{H})$ of organ $\mathrm{i}$

$$
D_{i}=\int D(t) f_{i}(t) d t, H_{i}=\int H(t) f_{i}(t) d t
$$

Determine the ESM R parameter

$$
D\left(R_{i}^{D}\right)=D_{i}, H\left(R_{i}^{H}\right)=H_{i}
$$

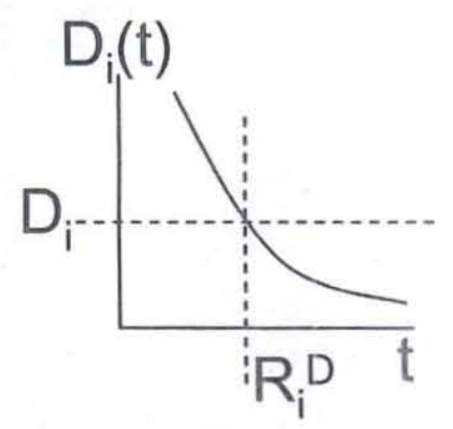




\section{Cumulative thickness distributions from CAM}

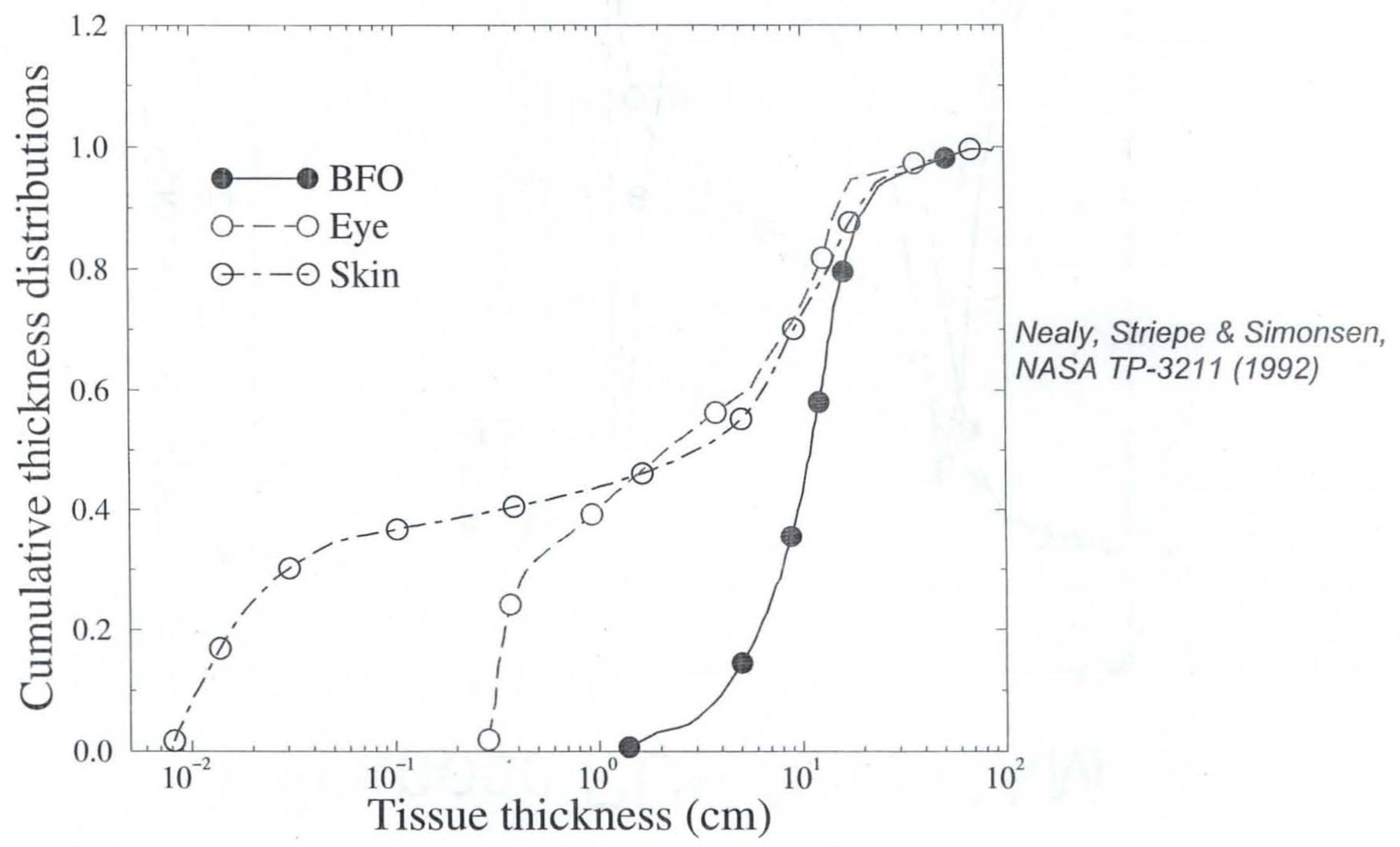




\section{Thickness PDF from CAM}

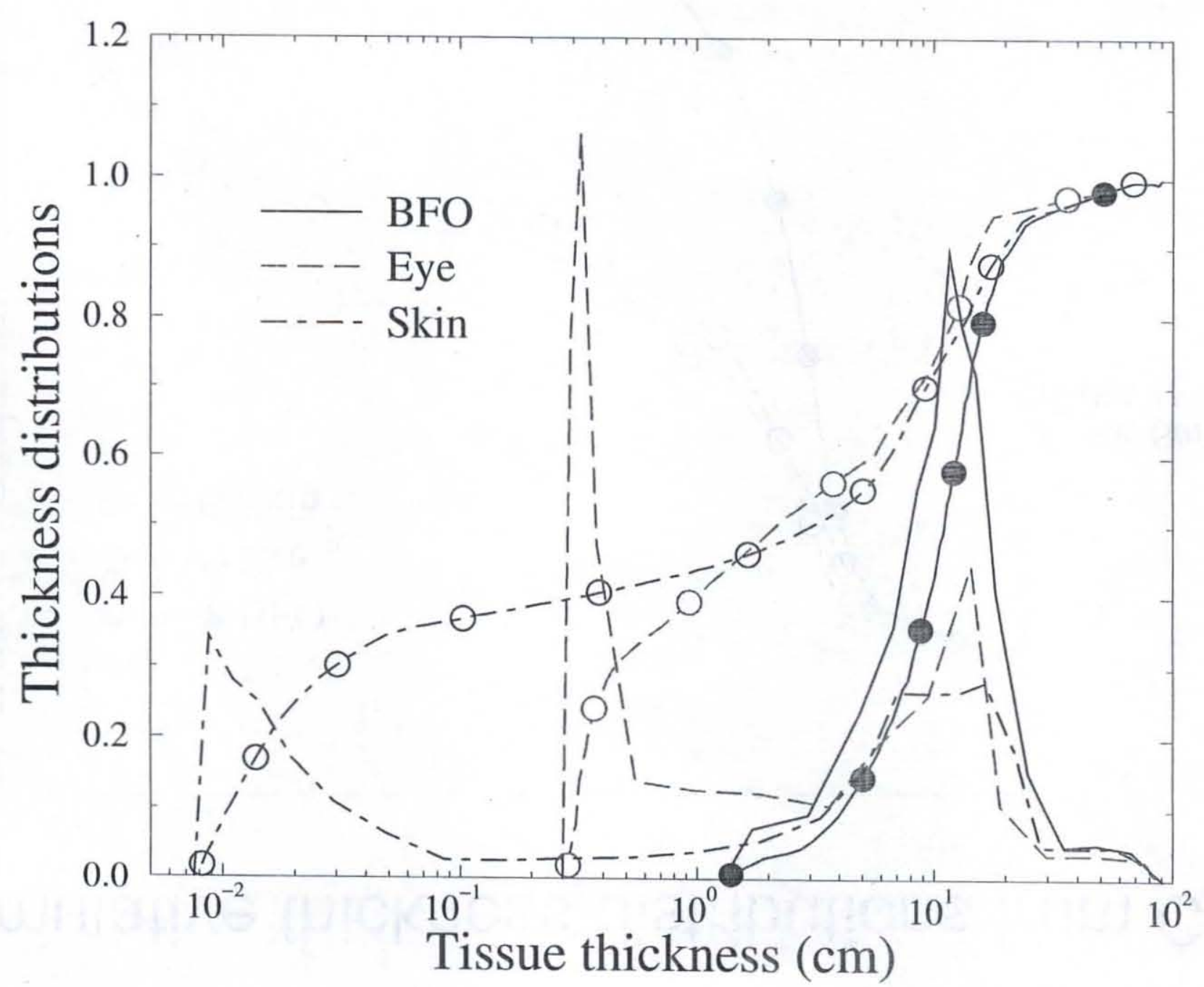

June 24-28, 2007

ANS/Space Nuclear Conference, Boston, MA 


\section{Dose-depth curves for 6 SPEs}

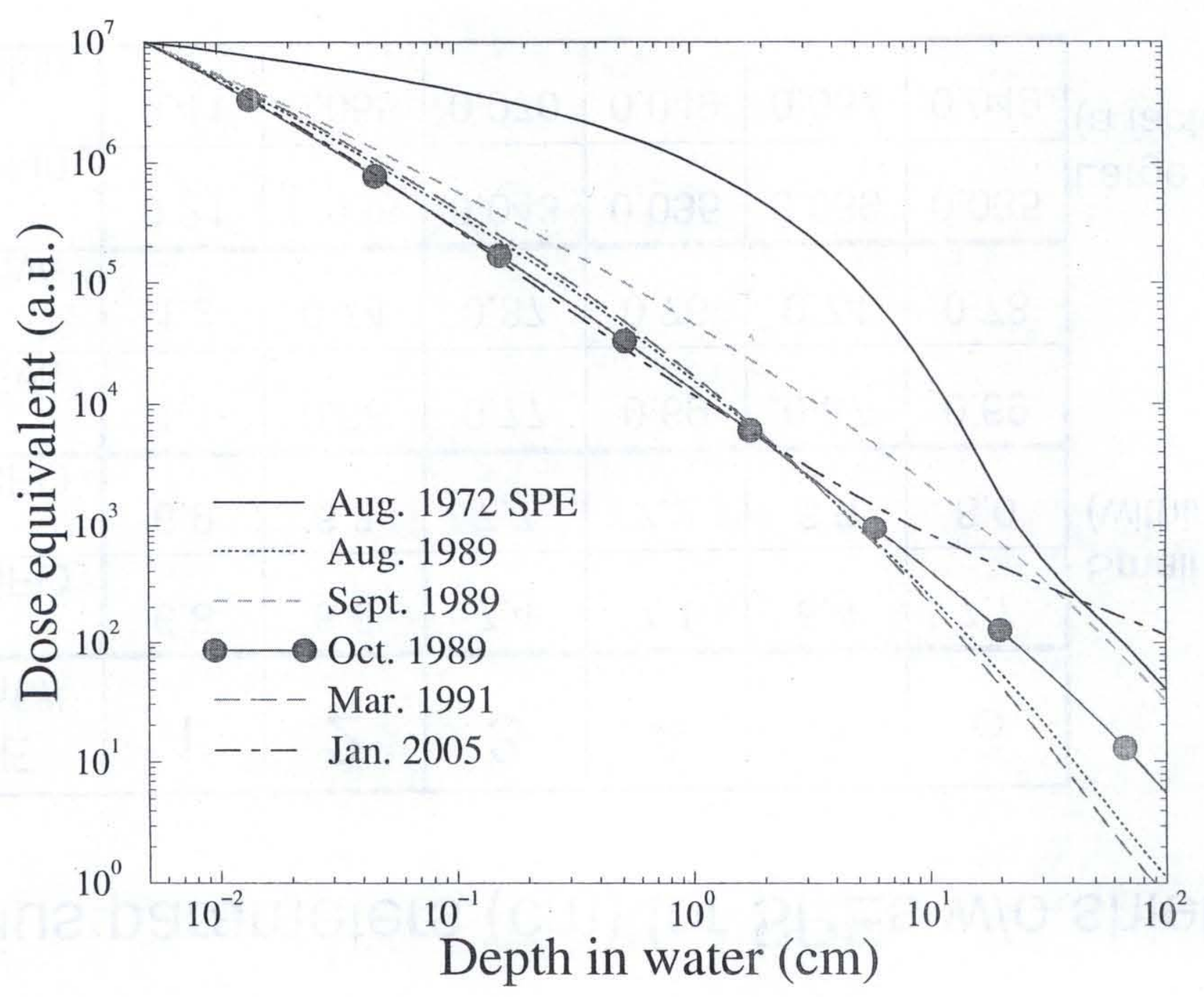




\section{Radius parameters (cm) for SPEs w/o shielding}

\begin{tabular}{|c|c|c|c|c|c|c|c|}
\hline $\begin{array}{c}\text { SPE } \\
\text { Event \# }\end{array}$ & 1 & 2 & 3 & 4 & 5 & 6 & \multirow{7}{*}{$\begin{array}{l}\text { Small variations } \\
\text { (within } 25 \% \text { ) }\end{array}$} \\
\hline $\mathrm{R}_{\mathrm{BFO}}$ & 6.8 & 6.6 & 7.4 & 7.1 & 6.4 & 7.7 & \\
\hline $\mathrm{R}_{\mathrm{BFO}}$ & 6.9 & 6.8 & 7.7 & 7.2 & 6.6 & 8.0 & \\
\hline $\mathrm{R}_{\text {Eye }}$ & 1.1 & 0.68 & 0.77 & 0.69 & 0.67 & 0.69 & \\
\hline $\mathrm{R}_{\text {Eye }}$ & 1.2 & 0.74 & 0.87 & 0.76 & 0.74 & 0.78 & \\
\hline $\mathrm{R}_{\text {Skin }}^{\mathrm{H}_{1}}$ & 0.21 & 0.038 & 0.043 & 0.035 & 0.039 & 0.035 & \\
\hline $\mathrm{R}_{\text {Skin }}$ & 0.41 & 0.055 & 0.070 & 0.049 & 0.057 & 0.049 & \\
\hline
\end{tabular}




\section{Dose-depth curves for 7 GCRs}

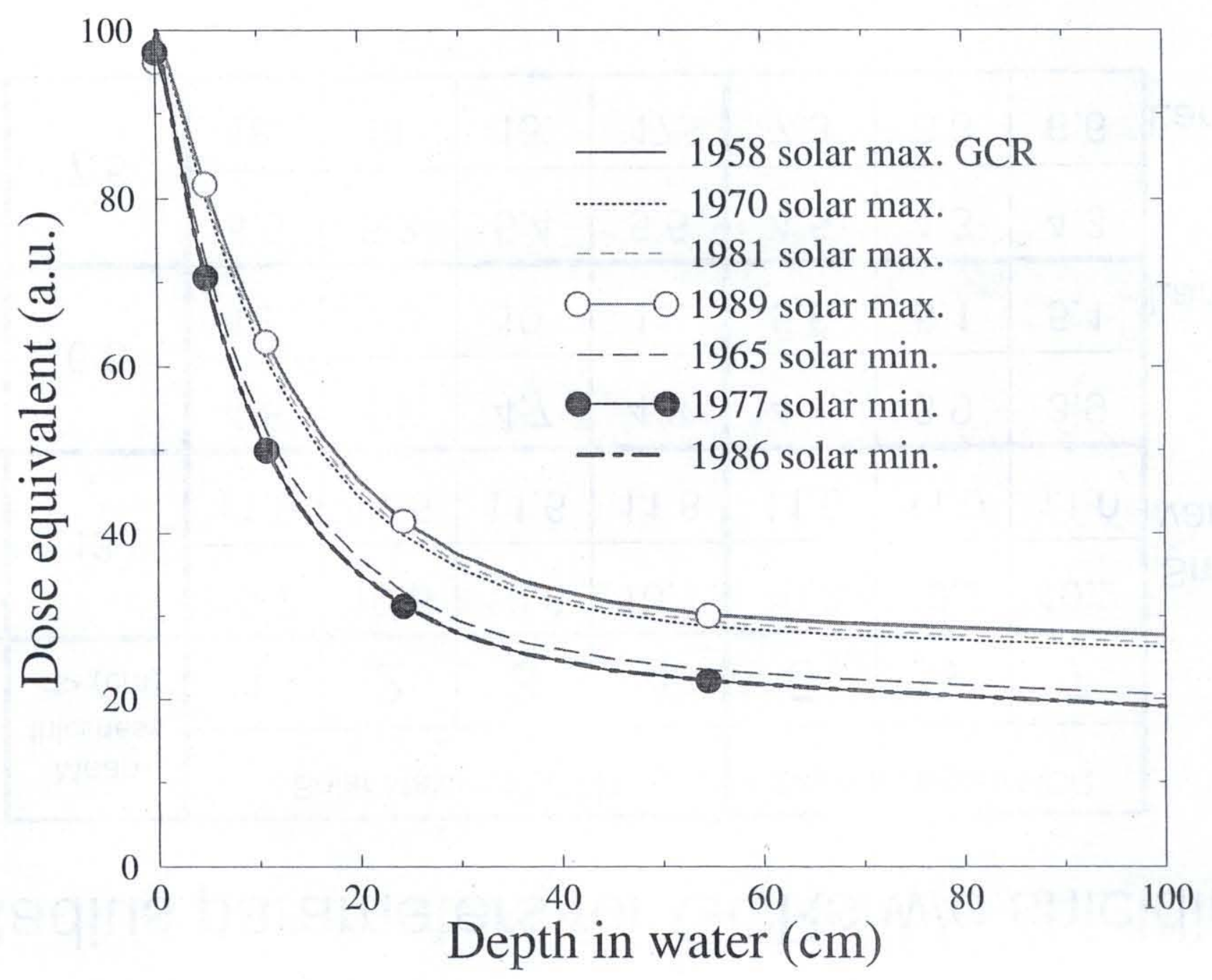

June 24-28, 2007

ANS/Space Nuclear Conference, Boston, MA 


\section{Radius parameters for GCRs w/o shielding}

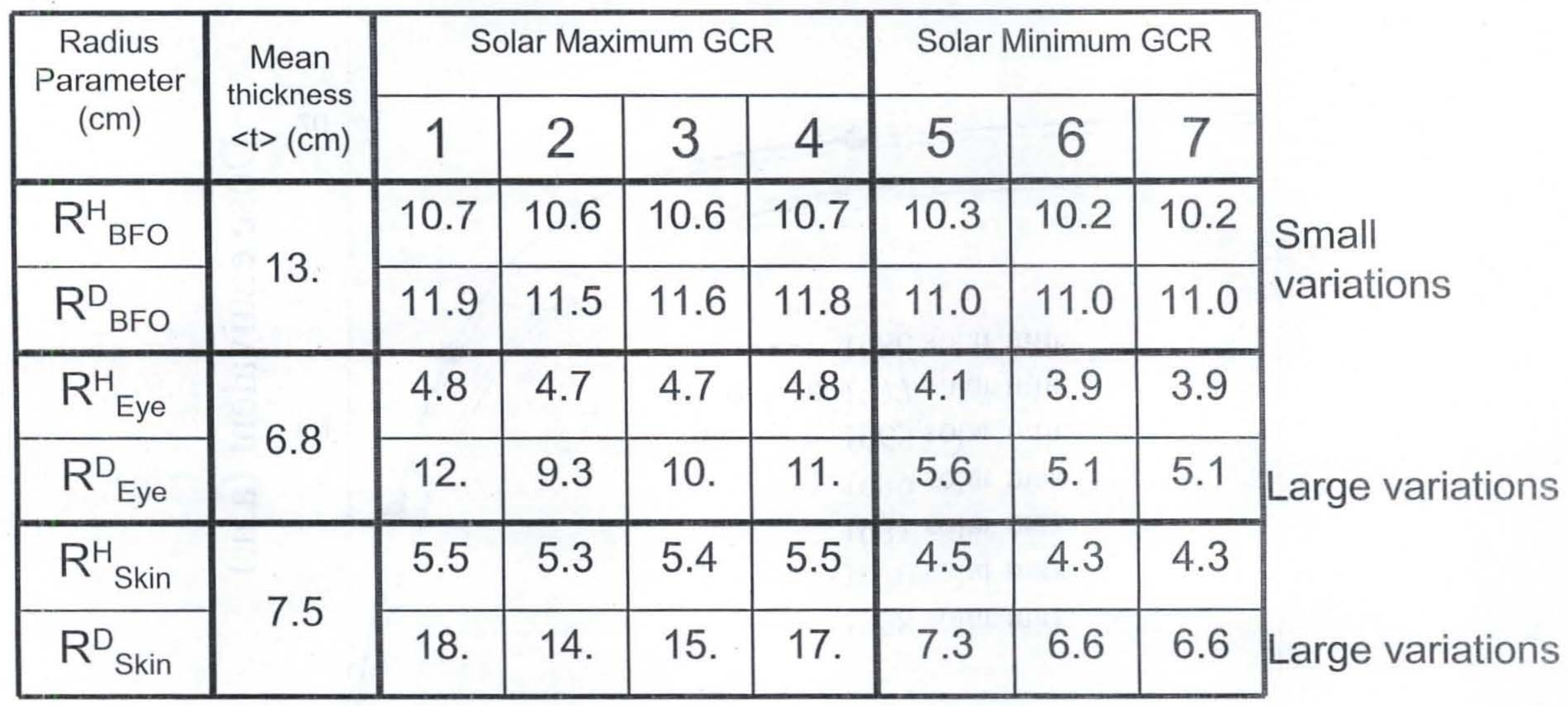




\section{Jansen's Inequality}

For a real convex function $\phi(x)$,

$$
\phi(E\{x\}) \leq E\{\phi(x)\}
$$

$E\{\phi(x)\}$ : the expectation value of $\phi(x)$ w.r.t. a PDF in $\mathrm{x}$

$$
\begin{aligned}
& \phi(x) \rightarrow H(t) \\
\Rightarrow & H\left(\left\langle t_{i}\right\rangle\right) \leq H_{i} \\
\Rightarrow & R_{i} \leq\left\langle t_{i}\right\rangle
\end{aligned}
$$

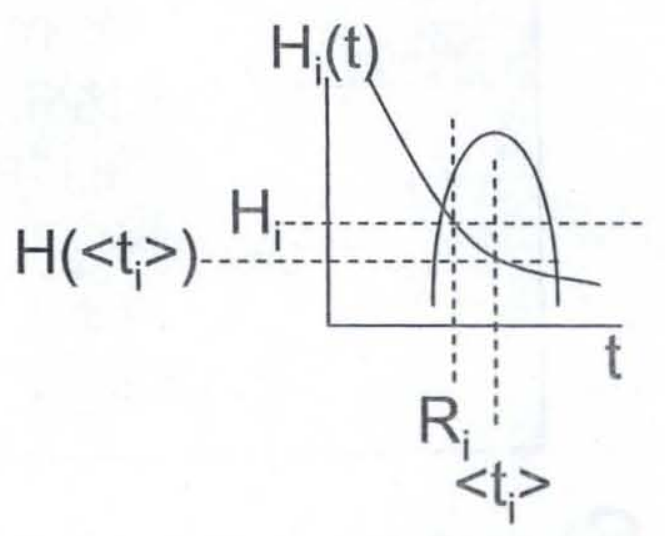




\section{$H(t)$ for SPEs with shielding}

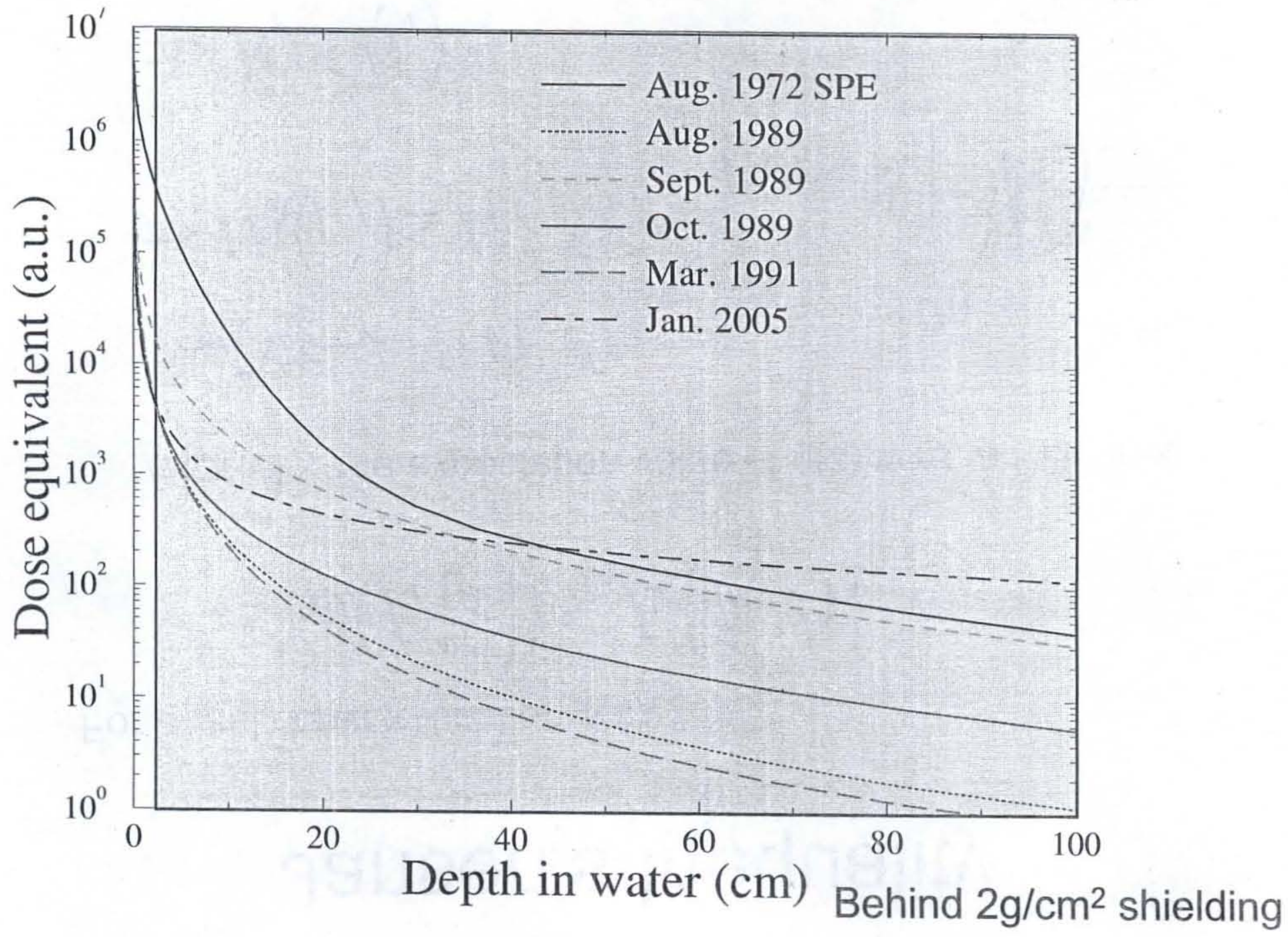




\section{$\mathrm{R}_{\mathrm{BFO}}$ for SPEs with shielding}

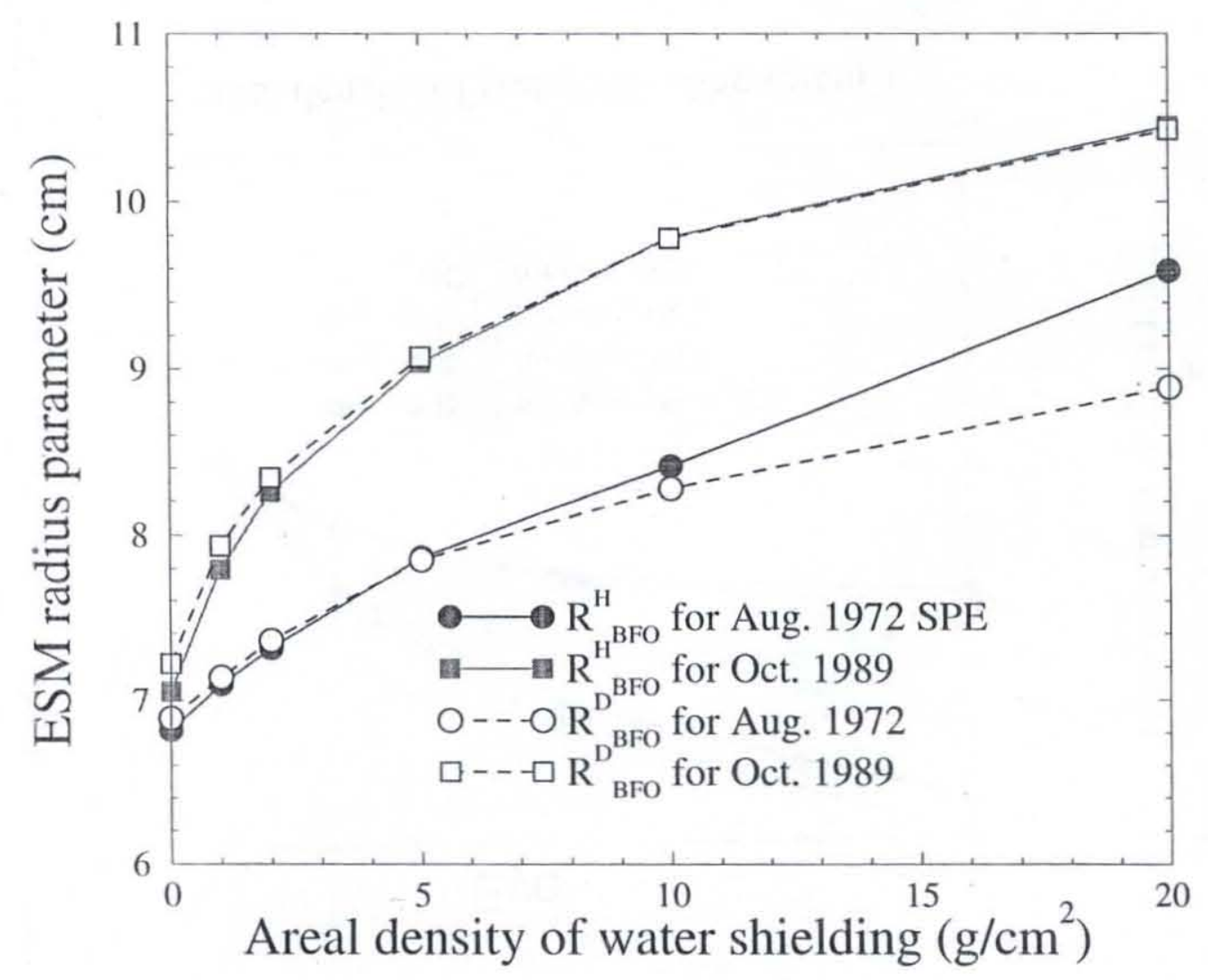

Moderate increase with shielding thickness

$\mathrm{R} \sim 7-11 \mathrm{fm}$

Consistent with the $9 \mathrm{~cm}$ sphere suggested for SPEs Bier, Townsend and Maxson, Adv. Space Res. 21 (1998) 


\section{For SPEs with shielding}
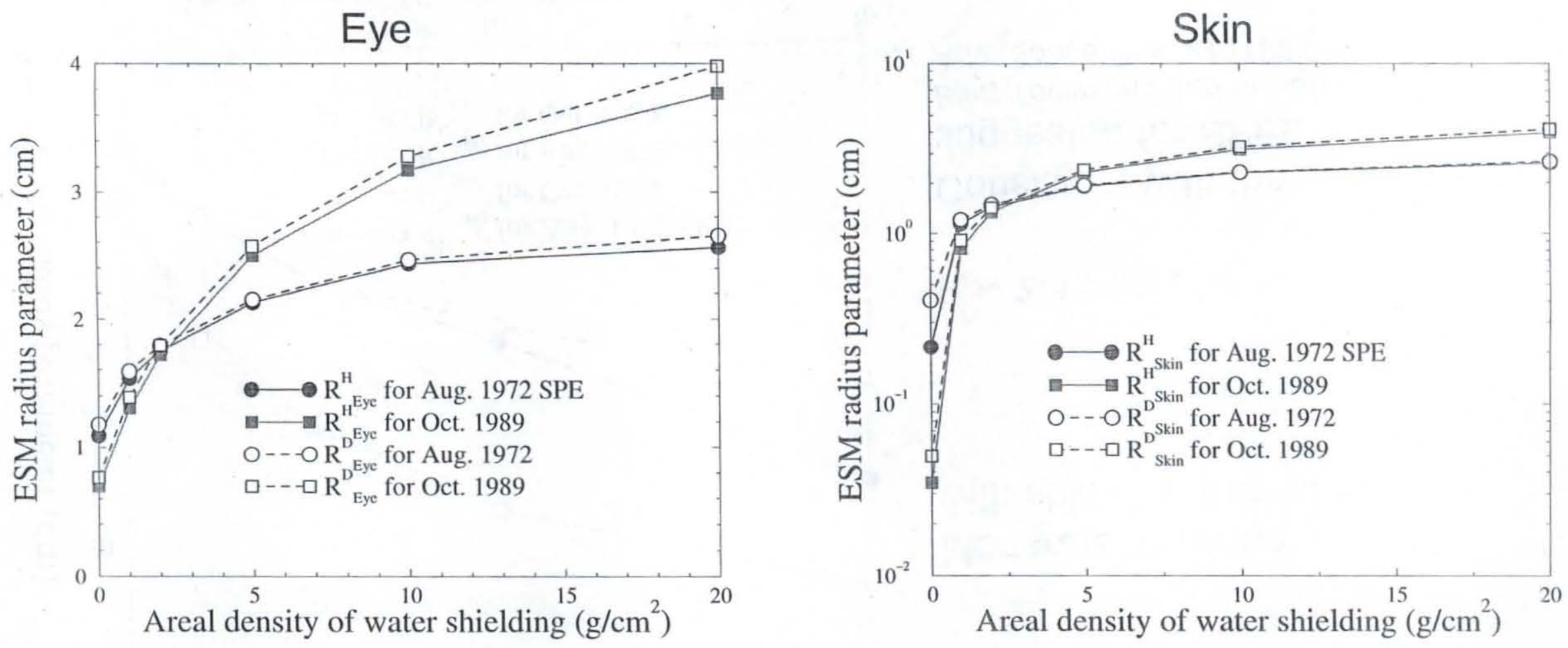

up to $\times 5$

up to $\times 100$

Strong increase with shielding thickness

$\Rightarrow$ ESM does not work here 


\section{$H(t)$ for GCRs with shielding}

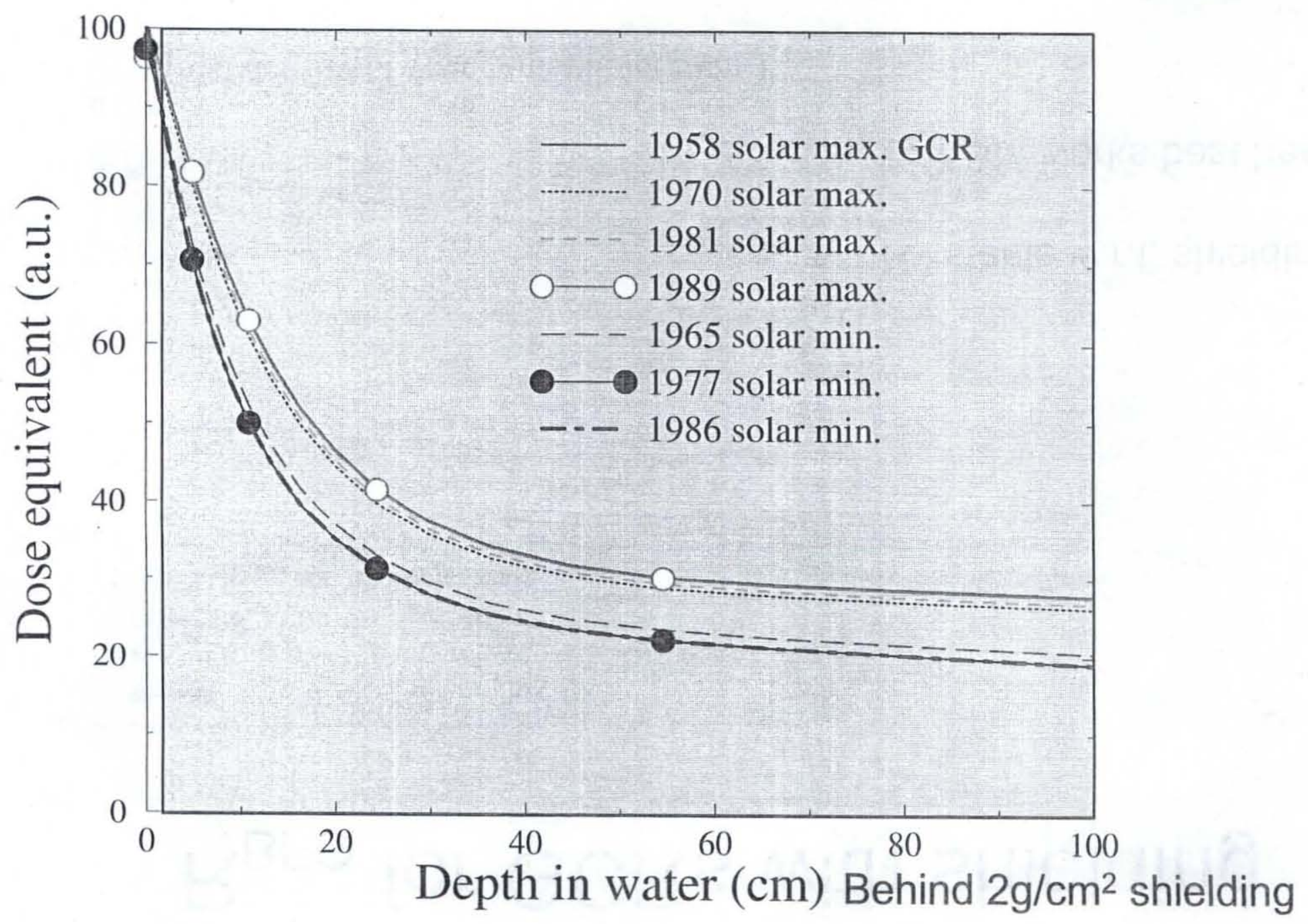




\section{$\mathrm{R}_{\mathrm{BFO}}$ for GCRs with shielding}

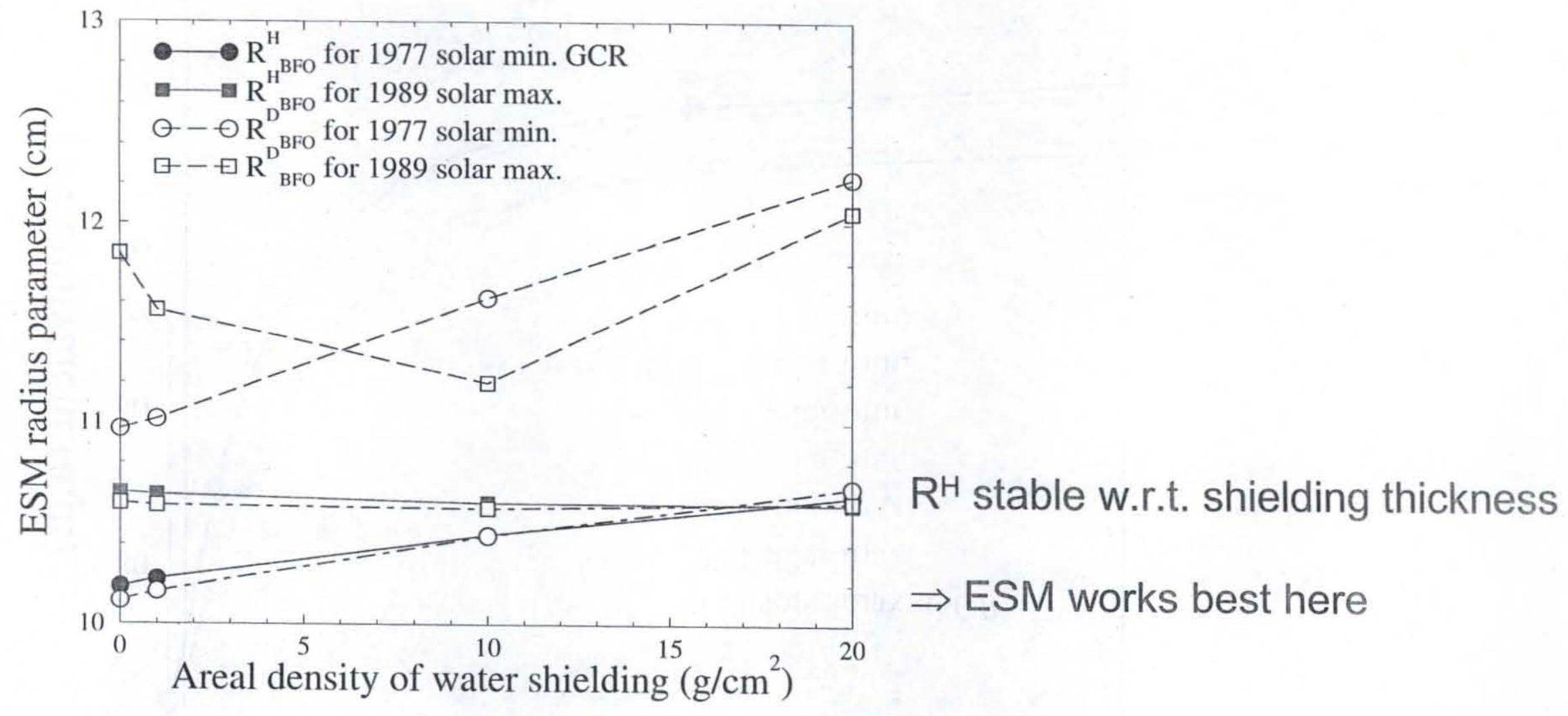




\section{For GCRs with shielding}
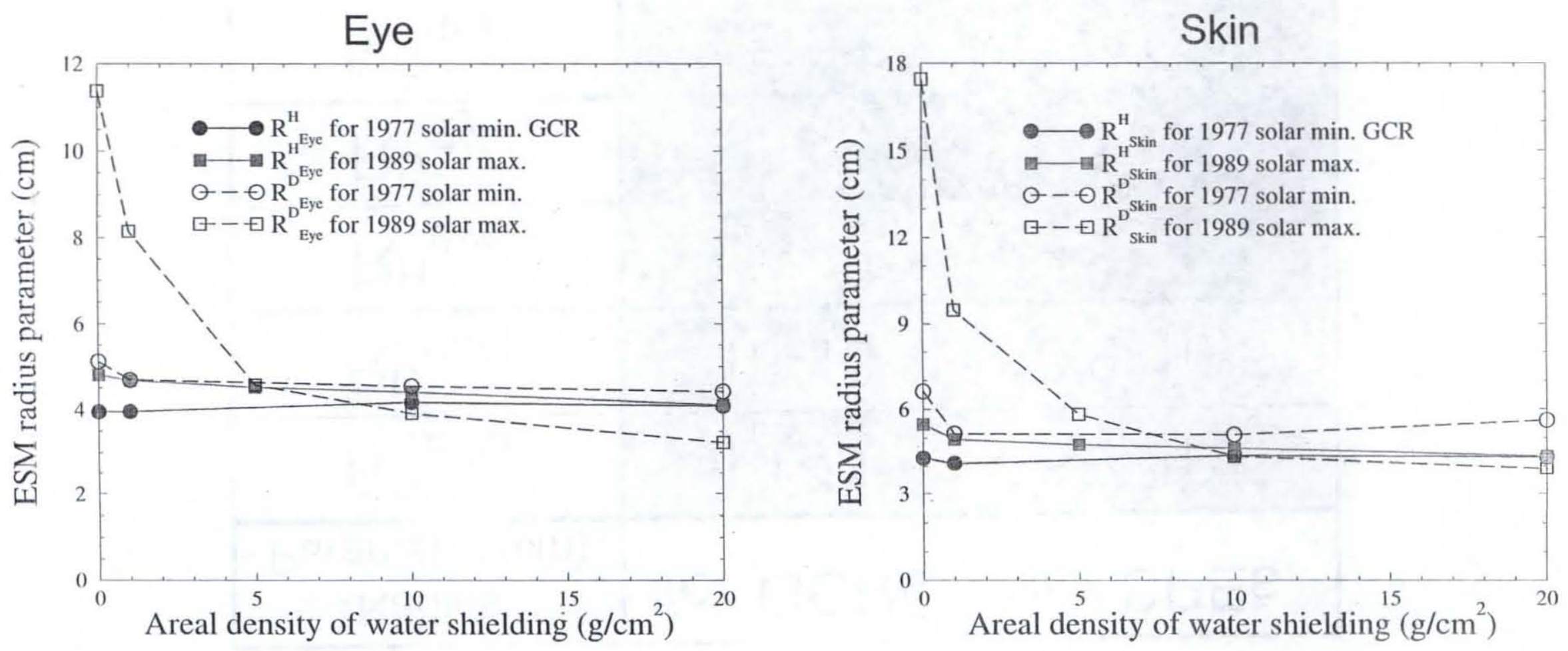

$\mathrm{R}^{\mathrm{H}}$ change moderately $\Rightarrow \mathrm{ESM}$ is marginal for $\mathrm{R}^{\mathrm{H}}$

$R^{D}$ changes sharply with shielding thickness $\Rightarrow E S M$ does not work for $R^{D}$ 


\section{ESM Goodness Matrix}

Color coding: Good, Marginal, Bad

\begin{tabular}{|c|c|c|}
\hline $\begin{array}{c}\text { Radius } \\
\text { Parameter (cm) }\end{array}$ & for GCRs & for SPEs \\
\hline $\mathrm{R}_{\mathrm{BFO}}^{\mathrm{H}}$ & $10-11$ & $6.8-10.5$ \\
\hline $\mathrm{R}_{\text {BFO }}$ & $10-13$ & $6.9-10.5$ \\
\hline $\mathrm{R}_{\text {Eye }}^{\mathrm{H}}$ & $3.7-4.8$ & $0.69-3.8$ \\
\hline $\mathrm{R}_{\text {Eye }}$ & $2.8-12$ & $0.76-4.0$ \\
\hline $\mathrm{R}_{\text {Skin }}$ & $3.5-5.6$ & $0.035-4.0$ \\
\hline $\mathrm{R}_{\text {Skin }}$ & $2.3-19$ & $0.049-4.2$ \\
\hline
\end{tabular}




\section{Summary}

Organ doses evaluated for water or Aluminum shielding from $t_{s}=0$ to $20 \mathrm{~g} / \mathrm{cm}^{2}$; Radius parameters from Equivalent Sphere Model (ESM) calculated.

\section{$\Rightarrow$ For SPEs:}

$\mathrm{R}_{\mathrm{BFO}} \sim 7-11 \mathrm{~cm}$, increases with $\mathrm{t}_{\mathrm{S}}$ by up to $50 \%$ (ESM marginal)

$R_{E y e} \& R_{\text {Skin }}$ increase sharply with $t_{S}$ (ESM does not work)

$\Rightarrow$ For GCRs:

$\mathrm{R}_{\mathrm{BFO}} \approx 10-11 \mathrm{~cm}$ (ESM works)

$\mathrm{R}_{\text {Eye }}^{\mathrm{H}} \sim 4-5 \mathrm{~cm}, \mathrm{R}_{\text {Skin }} \sim 3-6 \mathrm{~cm}, \mathrm{R}_{\text {BFO }} \sim 10-13 \mathrm{~cm}$ (ESM marginal)

$\mathrm{R}_{\text {Eye }}^{\mathrm{D}} \& \mathrm{R}_{\text {Skin }}$ change sharply with $\mathrm{t}_{\mathrm{S}}$ (ESM does not work) 